\title{
Myeloid deletion and therapeutic activation of AMP-activated protein kinase (AMPK) do not alter atherosclerosis in male or female mice
}

Nicholas D. LeBlond ${ }^{1,2,3}$, Peyman Ghorbani ${ }^{1,2,3}$, Conor O'Dwyer ${ }^{1,2,3}$, Nia Ambursley ${ }^{1}$, Julia R. C. Nunes ${ }^{1,2,3}$, Tyler K.T. Smith ${ }^{1,2,3}$, Natasha A.Trzaskalski ${ }^{1,2,4}$, Erin E. Mulvihill ${ }^{1,2,4}$, Benoit Viollet ${ }^{5}$, Marc Foretz ${ }^{5}$ and Morgan D. Fullerton ${ }^{1,2,3 *}$

${ }^{1}$ Department of Biochemistry, Microbiology and Immunology, Faculty of Medicine, University of Ottawa, Ottawa, ON, K1H 8M5, Canada

${ }^{2}$ Centre for Infection, Immunity and Inflammation, Ottawa ON K1H 8M5, Canada

${ }^{3}$ Centre for Catalysis Research and Innovation, Ottawa ON K1H 8M5, Canada

${ }^{4}$ University of Ottawa Heart Institute, Ottawa, ON K1Y 4W7, Canada

${ }^{5}$ Université de Paris, Institut Cochin, CNRS, INSERM, F-75014 Paris, France

*To whom correspondences should be addressed: Dr. Morgan Fullerton, Department of Biochemistry, Microbiology and Immunology, Faculty of Medicine, University of Ottawa, 4109A Roger Guindon Hall, 451 Smyth Rd, Ottawa, Ontario, Canada, K1H 8M5, Telephone (613) 562-5800 x8310; E-mail: morgan.fullerton@uottawa.ca

Running Title: Myeloid AMPK signaling in atherosclerosis

Keywords: AMP-activated protein kinase (AMPK), atherosclerosis, lipids, macrophage, immunometabolism. 


\section{Abstract:}

Objective: The dysregulation of myeloid-derived cell metabolism can drive atherosclerosis. AMP-activated protein kinase (AMPK) controls various aspects of macrophage dynamics and lipid homeostasis, which are important during atherogenesis.

Approach and Results: We aimed to clarify the role of myeloid-specific AMPK signaling by using LysM-Cre to drive the deletion of both the $\alpha 1$ and $\alpha 2$ catalytic subunits (MacKO), in male and female mice made acutely atherosclerotic by PCSK9AAV and Western diet-feeding. After 6 weeks of Western diet feeding, half received daily injection of either the AMPK activator, A-769662 or a vehicle control for a further 6 weeks. After 12 weeks, myeloid cell populations were not different between genotype or sex. Similarly, aortic sinus plaque size, lipid staining and necrotic area were not different in male and female MacKO mice compared to their littermate floxed controls. Moreover, therapeutic intervention with A-769662 had no effect. There were no differences in the amount of circulating total cholesterol or triglyceride, and only minor differences in the levels of inflammatory cytokines between groups. Finally, CD68+ area or markers of autophagy showed no effect of either lacking AMPK signaling or systemic AMPK activation.

\section{Conclusions:}

Our data suggest that while defined roles for each catalytic AMPK subunit have been identified, global deletion of myeloid AMPK signaling does not significantly impact atherosclerosis. Moreover, we show that intervention with the first-generation AMPK activator, A-769662, was not able to stem the progression of atherosclerosis. 


\section{Abbreviations}

ACC1/2

ALDH2

AMPK

CCR2

HDAC3

LC3 I/II

MacKO

MCP1

p62

WD

WT
Acetyl-CoA carboxylase 1 and 2

Aldehyde dehydrogenase 2

AMP-activated protein kinase

C-C chemokine receptor type 2

Histone deacetylase 3

Microtubule-associated proteins $1 \mathrm{~A} / 1 \mathrm{~B}$ light chain $3 \mathrm{~B}$

AMPKa1/a2 flox-LysM Cre+

Monocyte chemoattractant protein 1

Ubiquitin-binding protein p62 or Sequestosome-1

Western diet

Wild-type; AMPKa1/a2 flox-LysM Cre-

\section{Highlights:}

- The deletion of both catalytic subunits of AMPK in myeloid cells has no significant effect on the progression of atherosclerosis in either male or female mice

- Therapeutic delivery of a first-generation AMPK activator (A-769662) for the last 6 weeks of 12-week study had no beneficial effect in either male or female mice

- Studying total AMPK deletion may mask specific effects of each isoform and highlights the need for targeted disruption of AMPK phosphorylation sites via knock-in mutations, rather than the traditional "sledgehammer" knockout approach 


\section{Introduction:}

Atherosclerosis and its downstream cardiovascular complications continue to represent the leading cause of mortality and morbidity in developed countries. While the importance of cells such as vascular smooth muscle and adaptive immune cells in atherosclerosis has recently been highlighted, myeloid-derived cells of the innate immune system, monocytes and macrophages, are a primary driver of disease initiation and progression ${ }^{1,2}$. The haematopoietic differentiation of monocytes is crucial for atherogenesis, and it is now appreciated that modulating monocyte pools can have direct effects on atherosclerotic plaque initiation and progression ${ }^{3}$. Moreover, recent lines of evidence have pointed toward the intrinsic metabolic programming of myeloidderived cells as being a driver of their atherogenic and inflammatory potential ${ }^{4,5}$.

AMPK is an evolutionarily conserved heterotrimeric serine/threonine kinase that functions to maintain normal metabolic homeostasis by sensing and restoring energy deficits. AMPK acts to limit anabolic and stimulate catabolic programs in the cell. One of the most recognized consequences of AMPK activation is an acute inhibition of both fatty acid and cholesterol synthesis, via inhibiting phosphorylation on acetyl-CoA carboxylase 1 and 2 (ACC1 and 2) and 3-hydroxy-3-methyl-glutaryl-coenzyme A reductase, respectively ${ }^{6,7}$. The inhibition of $A C C$ results in the reduction of malonylCoA, which in addition to regulating lipogenic flux, relieves the inhibition on mitochondrial fatty acid uptake, leading to an increase in $\beta$-oxidation ${ }^{8}$. Complementary to this, AMPK, directly and indirectly, stimulates the process of macroautophagy (herein referred to as autophagy), which is critical for processing metabolic substrates, the 
clearance of damaged or senescent organelles and in the context of atherosclerosis, contributes to the mobilization of stored cholesterol in foam cells and reverse cholesterol transport ${ }^{9}$.

We and others have shown that AMPK signaling in differentiated cultured macrophages can regulate various aspects of fatty acid and cholesterol metabolism, while simultaneously governing broader metabolic and immune programs ${ }^{10-14}$. In the context of atherogenesis, there have been conflicting reports as to the role of myeloid AMPK signaling in the progression of atherosclerosis, though only male mice have been studied $^{15-17}$. Moreover, while systemic delivery of AMPK-activating treatments have been shown to reduce lesion size, the delivery of these treatments was over the entire course of the atherosclerosis progression model ${ }^{18,19}$ and the contribution of myeloid AMPK was not addressed. Here we report that in an acute model of atherogenesis instigated by AAV-delivery of a gain-of-function PCSK9, myeloid-specific disruption of both the $a 1$ and $a 2$ catalytic subunits of AMPK did not alter plaque size or necrotic core in male or female mice. Further to this, when the direct AMPK activating compound A769662 was administered to mice in a therapeutic, rather than preventative manner, no protection was observed. These unexpected findings lead us to question the specific roles of each catalytic subunit and whether novel allosteric activators of AMPK would offer therapeutic beneficial effects on atherogenesis.

\section{Materials and Methods:}

Materials and Methods are available in the supplementary material online. 


\section{Results:}

Deletion of myeloid AMPK signaling does not alter monocyte populations: While AMPKa1 is the predominant isoform expressed in haematopoietic cells ${ }^{12,20}$, myeloid deficiency of either AMPKa1 or AMPKa2 has been shown to alter atherosclerosis ${ }^{15-17}$. Moreover, these studies crossed their respective AMPK-deficient mouse models onto the atherogenic $L D L r$ - or ApoE-deficient background. To completely disrupt AMPK signaling, we generated mice lacking all AMPK signaling in myeloid cells (AMPKa1 and AMPKa2 deletion driven by LysM expression of Cre recombinase), which was confirmed by assessing AMPK-specific signaling to its downstream target ACC in elicited peritoneal macrophages from floxed littermate controls (wild-type; WT) and MacKO mice. As expected, basal AMPK signaling to ACC was almost completely absent in MacKO cells, with no effect of the AMPK activator, A-769662 (Supplemental Figure S1). As signaling to ACC has long been shown to be AMPK-specific ${ }^{21}$, the residual signal is likely due to the presence of non-myeloid cells. Importantly, basal AMPK signaling in the liver was unaffected (Supplemental Figure S1).

To circumvent genetic mouse models of atherosclerosis, we used a single intravenous injection of a well-characterized, gain-of-function (D377Y) mouse PCSK9AAV followed by Western diet (WD) feeding to induce rapid hypercholesterolemia to drive atherogenesis for 12 weeks (Supplemental Figure S2) ${ }^{22}$. Monocyte-derived macrophages are a key cell type in atherosclerosis, and myeloid cell maturation and differentiation, which begins in the bone marrow via myelopoiesis can be a driver of atherosclerosis. Moreover, myelopoiesis is augmented by WD-induced 
hypercholesterolemia in both mice and humans ${ }^{23}$. In a preliminary cohort of male and female WT and MacKO mice, we first aimed to determine if there were any basal genotype differences in myeloid cell populations. Using Ly6C, CD115 and Ly6G as myeloid cell markers, we observed no statistical differences in circulating, splenic or bone marrow populations, either between genotypes or between males and females (Figure 1A and Supplementary Figure S3). Moreover, the presence or absence of myeloid AMPK did not skew the levels of Ly6C $C^{\text {hi }}$ or Ly6C $C^{l o}$ populations, which are recognized as monocytes prone $\left(\right.$ Ly6C $\left.C^{\text {hi }}\right)$ or resistant $\left(\right.$ Ly6C $\left.^{l o}\right)$ to effector or patrolling functions, respectively ${ }^{2}$ (Figure $1 \mathrm{~B}$ and $\mathrm{C}$ ). To investigate myeloid differentiation, we determined that bone marrow $\mathrm{Lin}-\mathrm{Sca}-1^{+} \mathrm{C}-\mathrm{Kit}^{+}$cells, as well as bone marrow and splenic populations of multipotent progenitors, common myeloid progenitors, granulocyte-macrophage progenitors and megakaryocyte-erythroid progenitors were also unaltered (data not shown).

\section{Myeloid AMPK signaling has minimal impact on the atherosclerotic plaque: Male} and female mice of both genotypes gained weight as expected when fed a WD. After 6 weeks, each group was divided and received daily injections with either vehicle control or the first-generation AMPK activator A-769662 $(30 \mathrm{mg} / \mathrm{kg})$ for the last half of the 12week intervention. Both male and female mice, independent of myeloid AMPK signaling experienced a reduction in body weight, as has been previously documented ${ }^{24}$ (Supplemental Figure S4). At the completion of the 12-week study, the atherosclerotic lesion area was quantified from the aortic sinus. In male and female mice, there were no differences in lesion size between genotypes. In addition, treatment with A-769662 as 
an intervening therapy had no significant effect on plaque size (Figure 2A). When plaque sections were stained with Oil Red $O$ to quantify neutral lipid, there were no significant differences between groups (Figure 2B). Consistently, the amount of necrotic area was also not changed between by the presence of myeloid AMPK, treatment with A-769662 or between sex. (Figure 2C and Supplemental Figure S5). These data suggest that in the early stages of PCSK9-induced atherosclerosis, myeloid AMPK signaling does not regulate total lesion area, lipid content or necrotic area.

\section{Myeloid AMPK and systemic activation do not affect total circulating lipid levels:}

The liver is a significant regulator of whole-body lipid metabolism and can dictate levels of lipoprotein-associated cholesterol and triglyceride in the circulation. While disruption of AMPK was restricted to myeloid cells, there is the potential that LysM-mediated deletion would occur in liver-resident macrophages (Kupffer cells), which could affect circulating lipid levels ${ }^{25}$. Moreover, our experimental design made use of the systemic delivery of A-769662, which has well known effects on lipid metabolism ${ }^{21,24}$. Independent of genotype, treatment or sex, there were no differences in the levels of circulating total cholesterol or triglyceride (Figure $3 \mathrm{~A}$ and $\mathrm{B}$ ).

\section{Myeloid AMPK does not regulate systemic inflammation during atherosclerosis:}

Metabolism and inflammation are recognized as important drivers of atherogenesis. Given the known role for AMPK in modulating both macrophage metabolism and immune programs, we next assessed the systemic (circulating) levels of inflammatory cytokines (IL-10, IL-12p70, IL-6, KC, TNFa, IL-1ß, IFNy, IL-2, IL-4 and IL-5) (Figure 4 
and Supplemental Figure S6). In male mice, at the time of tissue harvest and blood collection, circulating levels of IL-12p70 and IL-4 were significantly lower in MacKO compared to control mice. When comparing vehicle and A-769662 treatment, IL-12p70 levels decreased in WT-treated but increased in MacKO-treated mice (Figure 3A-D). In addition, levels of KC (mouse IL-8), were significantly increased in A-769662-treated WT mice, but lower in MacKO mice treated with the AMPK activator. While levels of other cytokines went unchanged, IL-12p70, IL-4 and IL-10 were all augmented by A769662 treatment in mice that were deficient for myeloid AMPK, suggesting non-AMPK or non-myeloid mechanisms. In female animals, only IL-12p70 differed significantly, such that levels in MacKO mice were lower, but that also in WT mice, A-769662 treatment also caused a reduction in circulating levels.

Previous work has demonstrated that preventative AMPK activation (i.e. AMPK activator treatment at the start of dietary initiation of atherosclerosis) was associated with decreased levels of the circulating chemokine monocyte chemoattractant protein 1 $(\mathrm{MCP} 1)^{18,26}$. Consistent with a lack of difference in total plaque area, circulating MCP1 was not different between any of the groups (Figure S7).

Myeloid AMPK signaling does not alter the amount of CD68+ cells: Deletion of myeloid AMPK on an ApoE-deficient ${ }^{16}$ or $L D L r$-deficient ${ }^{17}$ background resulted in less and more markers of macrophage accumulation, respectively. To interrogate this in our model, we used CD68 as a general marker of macrophage-like cells within atherosclerotic lesions, since it is now well established that vascular smooth muscle cells can adopt CD68 expression as atherogenesis progresses ${ }^{27}$. Similar to results 
above, we observed no difference in aortic lesion area between male WT and MacKO, we detected no difference in the amount of lesion CD68+ expression between groups, regardless of genotype or treatment when normalized to total area (Figure $5 \mathrm{~A}$ and $\mathrm{B}$ ).

Myeloid AMPK signaling does not alter markers of autophagy: We next aimed to determine if the disruption or activation of AMPK signaling in myeloid cells influenced markers of autophagy within the plaque environment. We began by probing for the expression of autophagy markers Beclin-1 and microtubule-associated proteins $1 \mathrm{~A} / 1 \mathrm{~B}$ light chain 3B (LC3II/I) in whole aortic lysates of male WT and MacKO mice; however, no basal genotype differences were observed (Figure 6A). We next stained the atherosclerotic lesion for p62 (also known as SQSTM1), an essential chaperone protein that is processed in an autophagic-dependent matter and serves as a marker of defective autophagy ${ }^{28}$. The average level of plaque-associated p62 was unaffected by the presence or absence of myeloid AMPK, and treatment with A-769662 had no effect on the levels of p62 staining within lesions (Figure 6B and C) in male mice. We also observed that the localization of p62 staining within the plaque, which has been linked to its potential sequestration along with its cargo to autophagosomes, was also not regulated by genotype or treatment. 


\section{Discussion:}

The progression of atherosclerosis is causative in the development of cardiovascular disease. While mechanistic understanding has translated to effective frontline therapies against cardiovascular disease, incidence and societal burden remain high. Numerous pathways and cell types are engaged at the onset of atherosclerosis; however, among the complexity, metabolic and cell differentiation pathways of myeloid cells are critical ${ }^{2}$. Since AMPK is known to regulate multiple immunometabolic programs ${ }^{8,29}$, we sought to address the importance of AMPK signaling in myeloid cells during the progression of atherosclerosis. Moreover, building on evidence that chronic, preventative treatment of WD-fed ApoE-deficient mice with AMPK activators (including A-769662) protected against lesion development ${ }^{18,19}$, we asked whether therapeutic intervention with systemic AMPK activation protects against atherogenesis in a myeloid AMPK-dependent manner.

To avoid lengthy breeding schemes and the constitutive nature of $L d l r^{-/}$and $A p o E^{-/-}$mouse models, we administered a validated gain-of-function (D377Y) PCSK9AAV, which was driven by a liver-specific promoter ${ }^{22}$. While we aimed to provide clarity as to the role of myeloid AMPK in atherosclerosis, in our model, there were no statistical differences in plaque, lipid or necrotic area between 1) mice that had or did not have myeloid AMPK signaling, or 2) mice that were treated with or without the direct AMPK activator A-769662, in male and female mice. However, we observed that in male mice only, myeloid AMPK signaling showed a trend toward restraining the lipid content of atherosclerotic plaque in an AMPK-dependent manner (Figure 2B). This finding, though 
not statistically significant, is consistent with previous observations showing a protective role for AMPK signaling when the AMPKa1 subunit was deleted using LysM-Cre on an Ldlr-deficient background ${ }^{17}$. Similarly, we found that intervention with an AMPK activator tended to stem plaque lipid accumulation and that myeloid AMPK signaling seemed to be required.

There is no clear answer to the question as to whether AMPK and more specifically, myeloid AMPK plays a beneficial or detrimental role in atherogenesis. When myeloid AMPKa1 deletion was driven by LysM expression on a Ldlr-deficient background, these mice had more atherosclerotic lesion and plaque area, higher circulating lipids, more Ly $6 \mathrm{C}^{\text {hi }}$ monocytes and more pro-inflammatory markers in the aorta $^{17}$. Opposing this, myeloid AMPKa1 or AMPKa2 deletion via LysM-mediated Cre expression on an ApoE-deficient background resulted in smaller atherosclerotic lesions. This was attributed to an AMPKa1-dependent effect on regulating monocyte-tomacrophage differentiation and autophagy ${ }^{16}$ or an AMPKa2-dependent effect on DNA methylation and altered expression of matrix metalloproteinase ${ }^{15}$, respectively. The phenotype of the myeloid AMPKa2-deficient mice is interesting given that the predominant isoform in myeloid cells was shown to be AMPKa1 ${ }^{12,30}$. Given this, it might be expected that a compensatory up-regulation of the AMPKa2 subunit might occur during AMPKa1-deficiency; however, the opposite is not as intuitive.

Rather than deal with isoform-specific contributions, we chose to delete both catalytic subunits to best characterize the significance of the sum of myeloid AMPK signaling. One potential limitation to all studies that have used LysM-Cre as a driver of myeloid-specific disruption is the chronic and constitutive nature of the targeted 
deletion. However, even when this is considered, the largest discrepancy between studies that show a protective and those that show a detrimental role for myeloid AMPK is genetic background (LDLr vs. ApoE). Atherosclerosis is lessened in response to myeloid AMPKa1 and AMPKa2 deletion only when on an ApoE-deficient background ${ }^{15}$, ${ }^{16}$. Moreover, AMPKa1/ApoE mice had no difference in serum cholesterol and AMPKa2/ApoE mice had higher total cholesterol levels compared to floxed/ApoE control mice, suggesting that these effects were independent of circulating cholesterol. Contrary to this, circulating cholesterol levels in AMPKa1/LDLr mice were positively correlated with lesion size and were increased when myeloid AMPKa1 was absent ${ }^{17}$. We did not observe any differences in total levels of circulating lipids, which was consistent with the similarity in total lesion area in all groups. Yet lesion lipid accumulation in male myeloid AMPK-deficient mice trended toward being higher in our PCSK9-induced model (Figure 2B).

There remains the potential that regulatory differences in AMPK signaling exist in myeloid cells that have or do not have LDLr or ApoE. Aldehyde dehydrogenase 2 (ALDH2) disruption was atheroprotective on an ApoE-deficient background but atherogenic on a Ldlr-deficient background ${ }^{31}$. The presence of the LDLr blocks AMPK from phosphorylating ALDH2, which leads to histone deacetylase 3 (HDAC3)-mediated down-regulation of lysosomal programs and increased foam cell formation ${ }^{31}$. Two considerations are important when applying this to our current findings. First, myeloid deletion of AMPK signaling would remove the negative signaling to HDAC3 and serve to enhance programs that lower atherogenesis. Secondly, in our PCSK9-induced model, whether circulating PCSK9 has LDLr-suppressive effects in non-hepatic tissues 
(specifically circulating monocytes and plaque macrophages) remains unknown, making any interpretation of myeloid AMPK signaling working via LDLr premature.

AMPK signaling has been shown to induce autophagy directly via activating phosphorylation of Unc-51 like autophagy activating kinase 1, Beclin-1 and VPS34, and indirectly via inhibition of the mechanistic target of rapamycin complex 1 , which itself is regulated indirectly by AMPK-mediated phosphorylation of tuberous sclerosis complex 2 and/or regulatory-associated protein of $\mathrm{mTOR}^{32}$. It is now well established that autophagy is athero-protective due to its clearance of cellular debris, sequestration of defective organelles and liberation of free cholesterol for the purpose of cholesterol efflux $^{33}$. To assess one marker of lesion autophagy, we stained lesions for p62, the accumulation of which is associated with defective autophagy. Despite initial predictions, there were no genotype or treatment effects on the expression or localization p62 within the developed lesions. With a trending decrease in lesion lipid content, it remains entirely plausible that the effects of AMPK signaling are linked to its regulation of autophagy; however, this was not captured by p62 staining. In isolated macrophages, we and others have demonstrated that in addition to an acute regulation of autophagy induction, AMPK exerts a level of transcriptional regulation via direct and indirect modulation of transcription factor EB and lysosomal programs ${ }^{34-36}$.

Previous reports show that genetic deletion of myeloid AMPK primes them toward a more pro-inflammatory phenotype, elevating proportions of Ly6C ${ }^{\text {hi }}$ to Ly6C ${ }^{\text {lo }}$ monocytes in the circulation or in the peritoneum in response to thioglycollate ${ }^{17}$. In keeping with this, mice treated systemically and chronically with A-769662 had a similar response and lower Ly6C ${ }^{\text {hi }}$ cells ${ }^{18}$. In the present study, with all AMPK signaling 
disrupted, we observed absolutely no effect on circulating, splenic or bone marrow populations of myeloid or precursor cells. This was consistent with the lack of difference regarding plaque size but differ with results from $A p o E$ and $L d l$-deficient models ${ }^{15-17}$. As a correlate of immune infiltration into the plaque, we used CD68 as a marker of macrophages and macrophage-like cells, although CD68 is also known to be expressed on dendritic cells, neutrophils and smooth muscle cells as well ${ }^{27}$. Unexpectedly, we observed no differences in the apparent proportion of lesion associated CD68+ cells. While chronic A-769662 treatment in ApoE-deficient mice resulted in lower Ly6C ${ }^{\text {hi }}$ monocytes in conjunction with decreased C-C chemokine receptor type 2 (Ccr2) expression, this was recently expanded by the observation that in response to acute fasting or acute (4 h) AMPK activation with A-769662, Ly6C ${ }^{\text {hi }}$ monocytes are dramatically reduced due to lower levels of MCP1, the ligand for $\mathrm{CCR}^{26}$. In our model of complete myeloid AMPK-deletion, we see that circulating MCP1 is not altered. Importantly, while the effects of acute fasting and AMPK activation were attributed to a hepatic AMPK signaling, we did not observe any differences in MCP1 levels following systemic AMPK activation.

There have been an impressive number of studies that have aimed to assess the contribution of AMPK toward atherosclerosis. Whole-body ${ }^{16,37,38}$, vascular smooth muscle $^{39,40}$, endothelial ${ }^{39,41}$, and myeloid-specific models on either ApoE- or Ldlrdeficient backgrounds ${ }^{15-17}$ have been used in conjunction with either AMPKa1 or AMPKa2 knockout models. Despite this, to our knowledge, female mice have rarely (if ever) been assessed. Our results suggest that while there are no significant differences between male and female MacKO mice and WT littermates, there is a potential sex- 
specific trend whereby AMPK signaling may act to lower lesion lipid content in male, but not female mice. Sex-specific responses will continue to be an essential consideration in any future work. Moreover, a potential interpretation could be that the deletion of both catalytic subunits of AMPK, the effects (beneficial or detrimental) observed in models of single AMPK deletion may be lost. This point highlights the inherent limitations in knockout models of important metabolic regulators and the need for more pointed models to tease out the importance of specific signaling nodes.

We feel it is important to address some of the limitations and caveats of our study. 1) We began by addressing how myeloid AMPK deficiency may alter immune populations; however, we did not assess mice following the intervention with A-769662. While this may have illuminated differences in monocyte differentiation, there were no statistical differences in atherosclerosis. 2) Also, we chose to inject our WT and MacKO mice with a gain-of-function PCSK9 AAV to induce atherosclerosis $\left(2 \times 10^{10}\right.$ viral genomes per mouse), which at this concentration when coupled with the WD-feeding, resulted in levels of circulating cholesterol and plaque sizes that were lower than what is typically observed in $L D L r$-deficient mice ${ }^{22}$. Though 12 weeks of atherosclerosis progression should be sufficient for genotype differences to emerge, it may be possible that differences could have been observed earlier or later. Moreover, although treatment with A-769662 daily during atherogenesis was shown to be protective, intervention at the same dose was mainly ineffective and suggests that AMPK-activation may be necessary from the start of atherogenesis to provide a therapeutic benefit. However, this does not rule out the possibility that second-generation allosteric AMPK activators (PF-739, PF-249 or MK-8722), which are more potent and specific AMPK activators, will 
not show a therapeutic benefit during an intervention. This will be an important consideration for future work.

Our study is the first to question the role of both AMPKa1 and AMPKa2 in myeloid cells and suggests that the presence and activation of myeloid AMPK signaling does not impact atherosclerotic plaque size in the aortic root, independent of sex. Importantly, there were no changes in myeloid cell numbers, circulating lipid levels or systemic inflammation. While we have taken yet another approach to assessing the contribution of AMPK to atherogenesis (PCSK9-induced atherosclerosis and deletion of both myeloid AMPKa1 and AMPKa2), future work should concentrate on dissecting the specific molecular and metabolic pathways by which this important regulator may act. Moving forward, targeted phosphorylation knock-in mouse models of known AMPK substrates will be the only way to specifically interrogate the mechanism(s) by which AMPK-regulated pathways like autophagy and lipid metabolism affect atherosclerosis and other cardiometabolic diseases. 
Acknowledgements: We would like to thank Xiaoling Zhao for her assistance with histology and Dr Katey Rayner for her guidance with aortic sectioning.

Sources of Funding: This work was supported by Project Grants from the Canadian Institutes of Health Research (CIHR) (PJT148634 to M.D.F and PJT156136 to E.E.M) and a CIHR New Investigator award (MSH141981 to M.D.F.), an Early Research Leadership Initiative from the Heart and Stroke Foundation of Canada and its partners (M.D.F), an Ontario Ministry of Research, Innovation and Science Early Researcher Award (M.D.F) and the Agence Nationale de la Recherche (grant ANR-17-CE15-0030MetaTreg to B.V). N.D.L, T.K.T.S and J.R.C.N were all supported by an Ontario Graduate Scholarship.

Disclosures: None. 


\section{References}

1. Moore KJ, Sheedy FJ, Fisher EA. Macrophages in atherosclerosis: A dynamic balance. Nature reviews. Immunology. 2013;13:709-721

2. Flynn MC, Pernes G, Lee MKS, Nagareddy PR, Murphy AJ. Monocytes, macrophages, and metabolic disease in atherosclerosis. Front Pharmacol. 2019;10:666

3. Murphy AJ, Tall AR. Disordered haematopoiesis and athero-thrombosis. European heart journal. 2016;37:1113-1121

4. Koelwyn GJ, Corr EM, Erbay E, Moore KJ. Regulation of macrophage immunometabolism in atherosclerosis. Nat Immunol. 2018;19:526-537

5. Artyomov MN, Sergushichev A, Schilling JD. Integrating immunometabolism and macrophage diversity. Semin Immunol. 2016;28:417-424

6. Carlson CA, Kim KH. Regulation of hepatic acetyl coenzyme a carboxylase by phosphorylation and dephosphorylation. The Journal of biological chemistry. 1973;248:378-380

7. Beg ZH, Allmann DW, Gibson DM. Modulation of 3-hydroxy-3-methylglutaryl coenzyme a reductase activity with camp and wth protein fractions of rat liver cytosol. Biochemical and biophysical research communications. 1973;54:1362-1369

8. Day EA, Ford RJ, Steinberg GR. Ampk as a therapeutic target for treating metabolic diseases. Trends Endocrinol Metab. 2017

9. Ouimet M, Franklin V, Mak E, Liao X, Tabas I, Marcel YL. Autophagy regulates cholesterol efflux from macrophage foam cells via lysosomal acid lipase. Cell metabolism. 2011;13:655-667

10. Ouimet M, Ediriweera HN, Gundra UM, Sheedy FJ, Ramkhelawon B, Hutchison SB, Rinehold K, van Solingen C, Fullerton MD, Cecchini K, Rayner KJ, Steinberg GR, Zamore PD, Fisher EA, Loke P, Moore KJ. Microrna-33-dependent regulation of macrophage metabolism directs immune cell polarization in atherosclerosis. The Journal of clinical investigation. 2015;125:4334-4348

11. Fullerton MD, Ford RJ, McGregor CP, LeBlond ND, Snider SA, Stypa SA, Day EA, Lhotak S, Schertzer JD, Austin RC, Kemp BE, Steinberg GR. Salicylate improves macrophage cholesterol homeostasis via activation of ampk. Journal of lipid research. 2015;56:1025-1033

12. Galic S, Fullerton MD, Schertzer JD, Sikkema S, Marcinko K, Walkley CR, Izon D, Honeyman J, Chen ZP, van Denderen BJ, Kemp BE, Steinberg GR. Hematopoietic ampk beta1 reduces mouse adipose tissue macrophage inflammation and insulin resistance in obesity. The Journal of clinical investigation. 2011;121:4903-4915

13. Kemmerer M, Wittig I, Richter F, Brune B, Namgaladze D. Ampk activates Ixralpha and abca1 expression in human macrophages. Int J Biochem Cell Biol. 2016;78:1-9

14. Namgaladze D, Brune B. Macrophage fatty acid oxidation and its roles in macrophage polarization and fatty acid-induced inflammation. Biochimica et biophysica acta. 2016;1861:1796-1807

15. Fisslthaler B, Zippel N, Abdel Malik R, Delgado Lagos F, Zukunft S, Thoele J, Siuda D, Soehnlein O, Wittig I, Heidler J, Weigert A, Fleming I. Myeloid-specific deletion of the ampkalpha2 subunit alters monocyte protein expression and atherogenesis. Int $J \mathrm{Mol}$ Sci. 2019;20

16. Zhang M, Zhu H, Ding Y, Liu Z, Cai Z, Zou MH. Amp-activated protein kinase alpha1 promotes atherogenesis by increasing monocyte-to-macrophage differentiation. The Journal of biological chemistry. 2017

17. Cao Q, Cui X, Wu R, Zha L, Wang X, Parks JS, Yu L, Shi H, Xue B. Myeloid deletion of alpha1ampk exacerbates atherosclerosis in low density lipoprotein receptor knockout (Idlrko) mice. Diabetes. 2016 
18. Wang J, Ma A, Zhao M, Zhu H. Ampk activation reduces the number of atheromata macrophages in apoe deficient mice. Atherosclerosis. 2017;258:97-107

19. Ma A, Wang J, Yang L, An Y, Zhu H. Ampk activation enhances the anti-atherogenic effects of high density lipoproteins in apoe(-/-) mice. Journal of lipid research. 2017;58:1536-1547

20. Mounier R, Theret M, Arnold L, Cuvellier S, Bultot L, Goransson O, Sanz N, Ferry A, Sakamoto K, Foretz M, Viollet B, Chazaud B. Ampkalpha1 regulates macrophage skewing at the time of resolution of inflammation during skeletal muscle regeneration. Cell metabolism. 2013;18:251-264

21. Fullerton MD, Galic S, Marcinko K, Sikkema S, Pulinilkunnil T, Chen ZP, O'Neill HM, Ford RJ, Palanivel R, O'Brien M, Hardie DG, Macaulay SL, Schertzer JD, Dyck JR, van Denderen BJ, Kemp BE, Steinberg GR. Single phosphorylation sites in acc1 and acc2 regulate lipid homeostasis and the insulin-sensitizing effects of metformin. Nature medicine. 2013;19:1649-1654

22. Bjorklund MM, Hollensen AK, Hagensen MK, Dagnaes-Hansen F, Christoffersen C, Mikkelsen JG, Bentzon JF. Induction of atherosclerosis in mice and hamsters without germline genetic engineering. Circulation research. 2014;114:1684-1689

23. Rahman MS, Murphy AJ, Woollard KJ. Effects of dyslipidaemia on monocyte production and function in cardiovascular disease. Nature reviews. Cardiology. 2017;14:387-400

24. Cool B, Zinker B, Chiou W, Kifle L, Cao N, Perham M, Dickinson R, Adler A, Gagne G, lyengar R, Zhao G, Marsh K, Kym P, Jung P, Camp HS, Frevert E. Identification and characterization of a small molecule ampk activator that treats key components of type 2 diabetes and the metabolic syndrome. Cell metabolism. 2006;3:403-416

25. Huang W, Metlakunta A, Dedousis N, Zhang P, Sipula I, Dube JJ, Scott DK, O'Doherty RM. Depletion of liver kupffer cells prevents the development of diet-induced hepatic steatosis and insulin resistance. Diabetes. 2010;59:347-357

26. Jordan S, Tung N, Casanova-Acebes M, Chang C, Cantoni C, Zhang D, Wirtz TH, Naik S, Rose SA, Brocker CN, Gainullina A, Hornburg D, Horng S, Maier BB, Cravedi P, LeRoith D, Gonzalez FJ, Meissner F, Ochando J, Rahman A, Chipuk JE, Artyomov MN, Frenette PS, Piccio L, Berres ML, Gallagher EJ, Merad M. Dietary intake regulates the circulating inflammatory monocyte pool. Cell. 2019;178:1102-1114 e1117

27. Dubland JA, Francis GA. So much cholesterol: The unrecognized importance of smooth muscle cells in atherosclerotic foam cell formation. Curr Opin Lipidol. 2016;27:155-161

28. Sergin I, Bhattacharya S, Emanuel R, Esen E, Stokes CJ, Evans TD, Arif B, Curci JA, Razani B. Inclusion bodies enriched for $\mathrm{p} 62$ and polyubiquitinated proteins in macrophages protect against atherosclerosis. Sci Signal. 2016;9:ra2

29. Fullerton MD, Steinberg GR, Schertzer JD. Immunometabolism of ampk in insulin resistance and atherosclerosis. Molecular and cellular endocrinology. 2013;366:224-234

30. Dong C, Zhao G, Zhong M, Yue Y, Wu L, Xiong S. Rna sequencing and transcriptomal analysis of human monocyte to macrophage differentiation. Gene. 2013;519:279-287

31. Zhong S, Li L, Zhang YL, Zhang L, Lu J, Guo S, Liang N, Ge J, Zhu M, Tao Y, Wu YC, Yin $\mathrm{H}$. Acetaldehyde dehydrogenase 2 interactions with Idlr and ampk regulate foam cell formation. The Journal of clinical investigation. 2019;129:252-267

32. Herzig S, Shaw RJ. Ampk: Guardian of metabolism and mitochondrial homeostasis. Nature reviews. Molecular cell biology. 2018;19:121-135

33. Ouimet M. Autophagy in obesity and atherosclerosis: Interrelationships between cholesterol homeostasis, lipoprotein metabolism and autophagy in macrophages and other systems. Biochimica et biophysica acta. 2013;1831:1124-1133

34. Young NP, Kamireddy A, Van Nostrand JL, Eichner LJ, Shokhirev MN, Dayn Y, Shaw RJ. Ampk governs lineage specification through tfeb-dependent regulation of lysosomes. Genes Dev. 2016;30:535-552 
35. El-Houjeiri L, Possik E, Vijayaraghavan T, Paquette M, Martina JA, Kazan JM, Ma EH, Jones R, Blanchette P, Puertollano R, Pause A. The transcription factors tfeb and tfe3 link the flcn-ampk signaling axis to innate immune response and pathogen resistance. Cell reports. 2019;26:3613-3628 e3616

36. Collodet C, Foretz M, Deak M, Bultot L, Metairon S, Viollet B, Lefebvre G, Raymond F, Parisi A, Civiletto G, Gut P, Descombes P, Sakamoto K. Ampk promotes induction of the tumor suppressor flcn through activation of tfeb independently of mtor. FASEB journal : official publication of the Federation of American Societies for Experimental Biology. 2019;33:12374-12391

37. Pinkosky SL, Newton RS, Day EA, Ford RJ, Lhotak S, Austin RC, Birch CM, Smith BK, Filippov S, Groot PH, Steinberg GR, Lalwani ND. Liver-specific atp-citrate lyase inhibition by bempedoic acid decreases Idl-c and attenuates atherosclerosis. Nat Commun. 2016;7:13457

38. Wang Q, Zhang M, Liang B, Shirwany N, Zhu Y, Zou MH. Activation of amp-activated protein kinase is required for berberine-induced reduction of atherosclerosis in mice: The role of uncoupling protein 2. PloS one. 2011;6:e25436

39. Cai Z, Ding Y, Zhang M, Lu Q, Wu S, Zhu H, Song P, Zou MH. Ablation of adenosine monophosphate-activated protein kinase alpha1 in vascular smooth muscle cells promotes diet-induced atherosclerotic calcification in vivo. Circulation research. 2016;119:422-433

40. Ding Y, Zhang M, Zhang W, Lu Q, Cai Z, Song P, Okon IS, Xiao L, Zou MH. Ampactivated protein kinase alpha 2 deletion induces vsmc phenotypic switching and reduces features of atherosclerotic plaque stability. Circulation research. 2016;119:718730

41. Dong Y, Zhang M, Wang S, Liang B, Zhao Z, Liu C, Wu M, Choi HC, Lyons TJ, Zou MH. Activation of amp-activated protein kinase inhibits oxidized Idl-triggered endoplasmic reticulum stress in vivo. Diabetes. 2010;59:1386-1396 
A

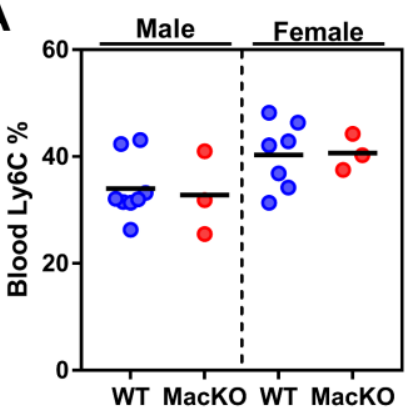

B

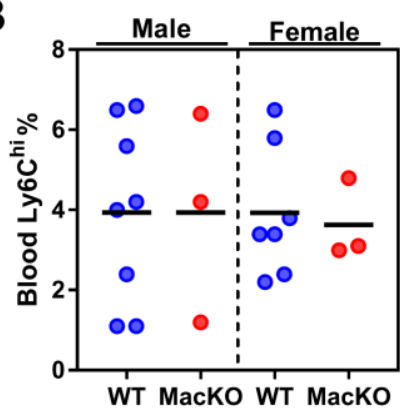

C

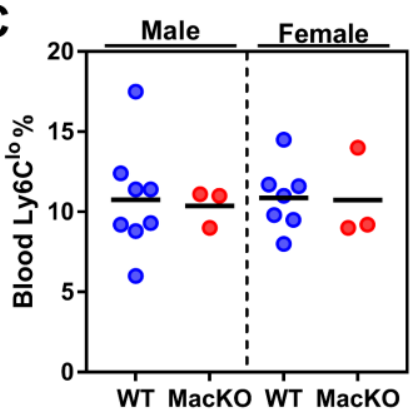

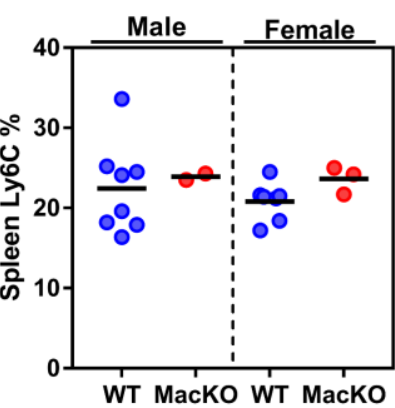
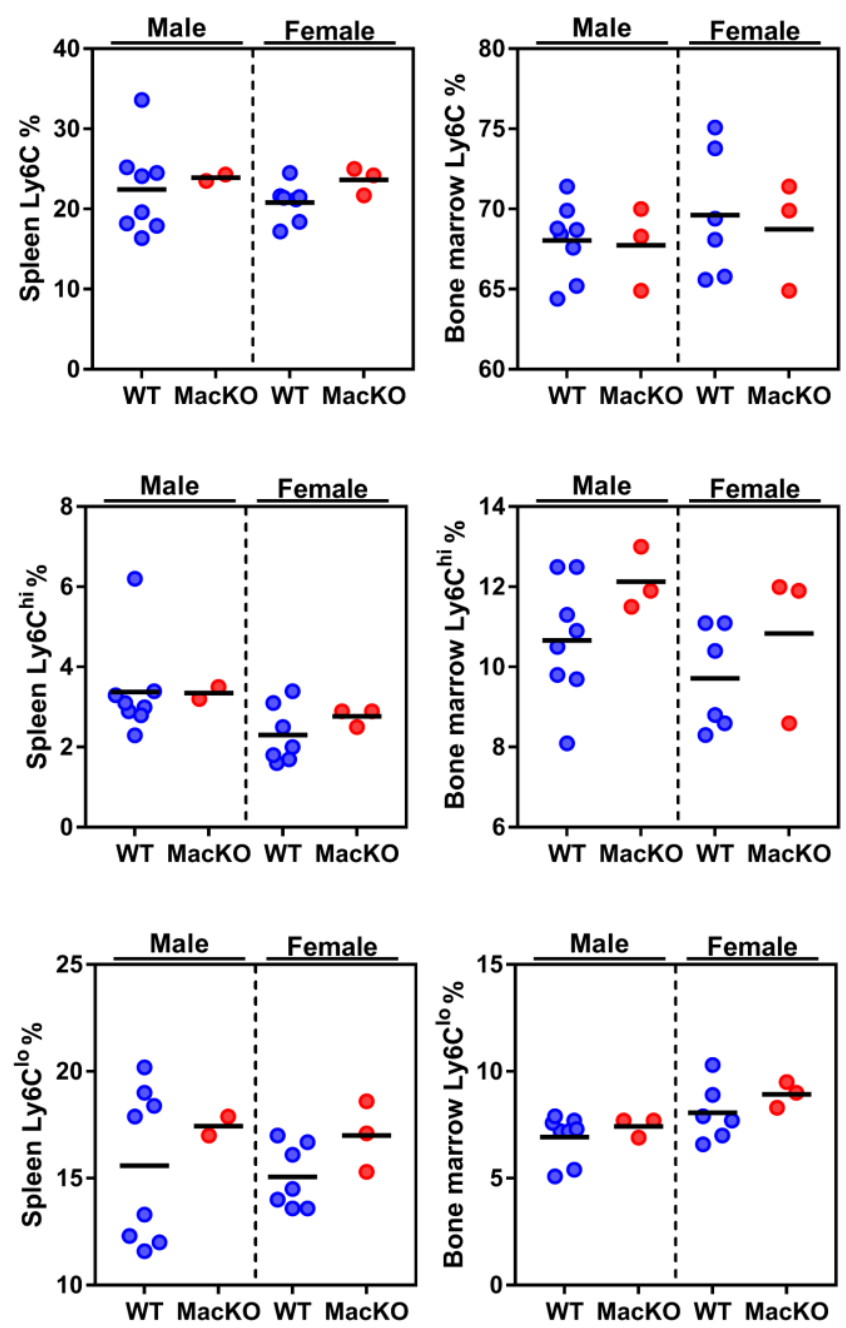

Figure 1. Myeloid AMPK does not alter myelopoiesis. WT and MacKO male and female mice were injected with the PCSK9-AAV and fed a WD for 12 weeks. The expression of $(A)$ total Ly6C, $(B)$ Ly6C hi and $(C)$ Ly6 $6 C^{l o}$ populations from the blood, spleen, and bone marrow quantified. Each data point represents the mean value from one animal ( $n=3-8 /$ group). 
A
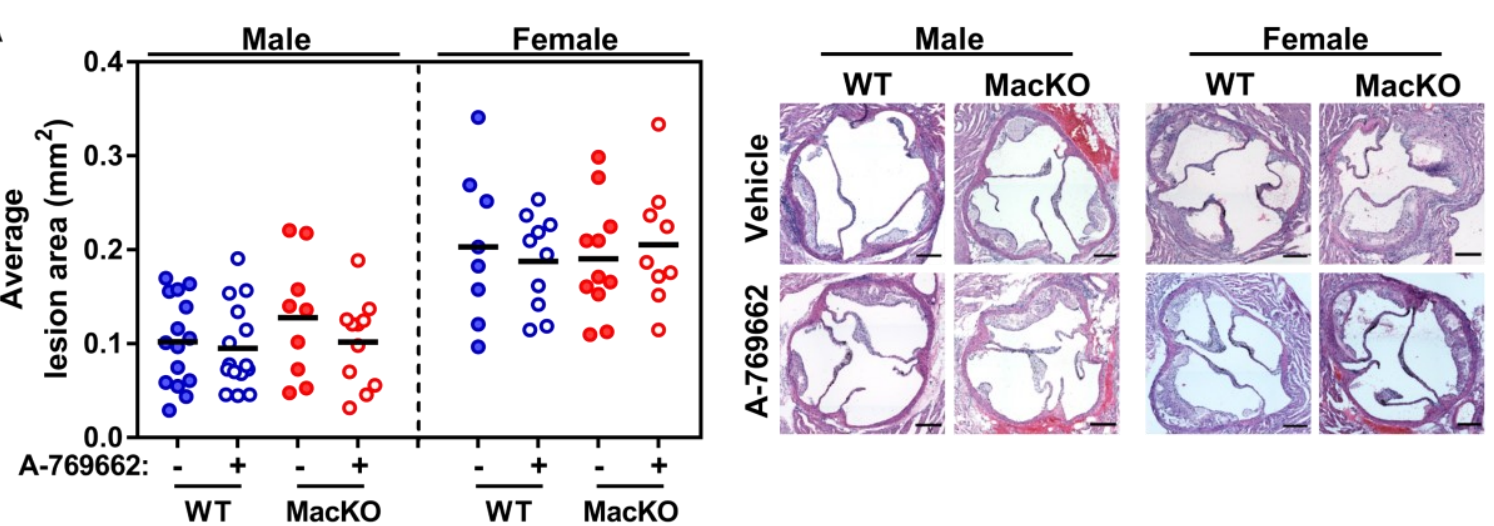

B
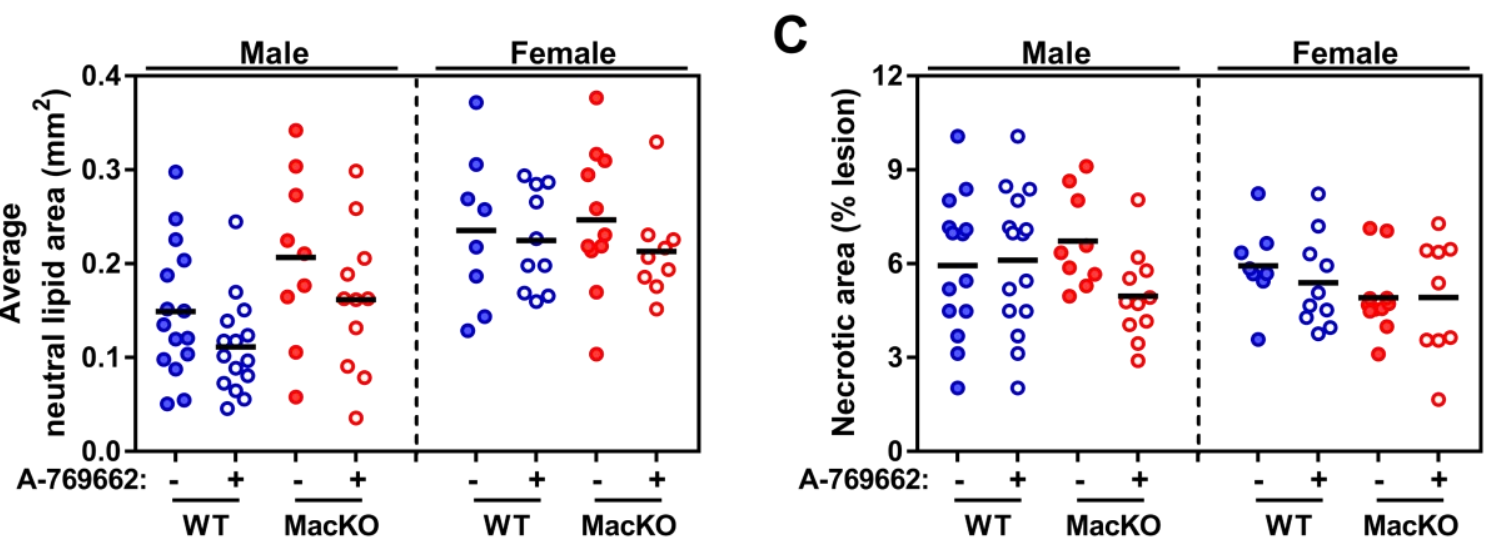

Figure 2. Myeloid AMPK signaling does not alter the progression of atherosclerosis in male and female mice. WT and MacKO male and female mice were injected with the PCSK9-AAV and fed a WD for 12 weeks, with half of each group receiving either $30 \mathrm{mg} / \mathrm{kg}$ A-769662 or vehicle control for the final 6 weeks. (A) Average lesion area quantified within the aortic sinus (representative H\&E-stained images are included). (B) Quantification of the average oil red $\mathrm{O}$ containing areas within the aortic sinus. (C) Lesion necrotic area expressed as percent lesion area. (A-C) Selection and quantifications were performed with Image $\mathrm{J}$ software of $(\mathrm{A}$ and $\mathrm{C}$ ) $\mathrm{H}$ and $\mathrm{E}$ and $(\mathrm{B})$ Oil Red $\mathrm{O}$ stained slides as listed in the materials and methods. Each data point represents the mean value from one animal ( $n=7$-16/group). 

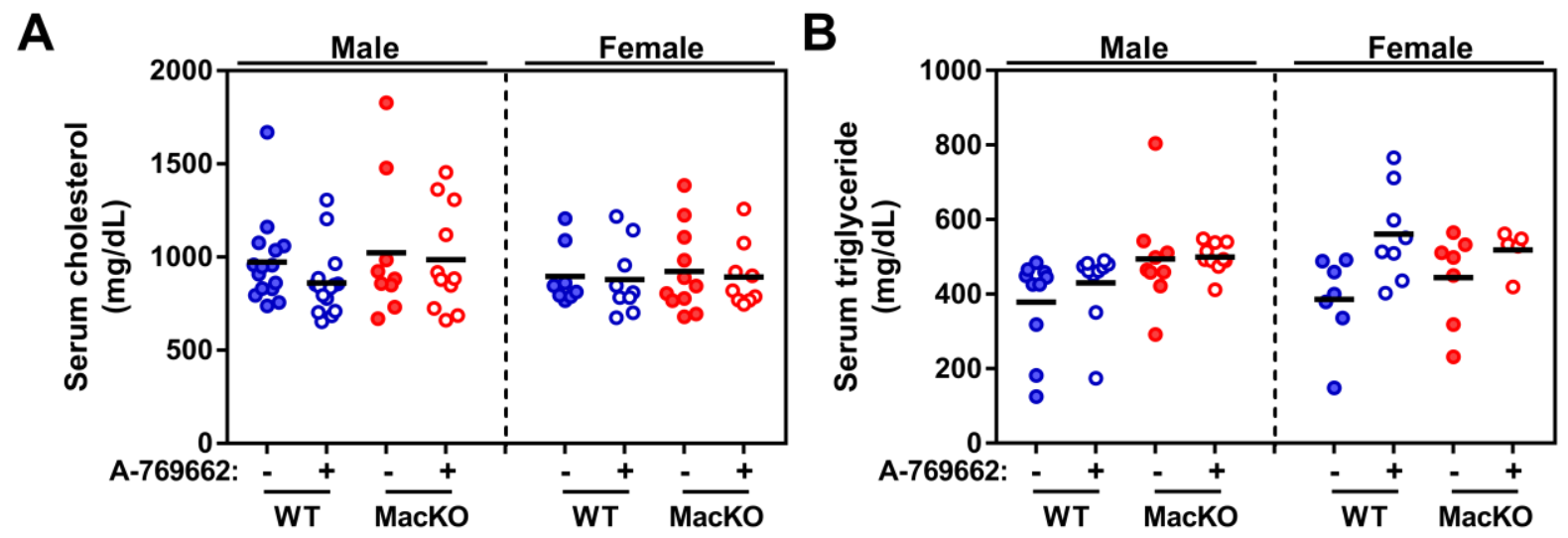

Figure 3. Myeloid AMPK signaling does not change total levels of circulating lipids. Following WD-feeding, serum was collected to assess (A) total circulating cholesterol and $(\mathrm{B})$ total circulating triglycerides. Each data point represents the value from one animal ( $n=5$-16/group). 
A

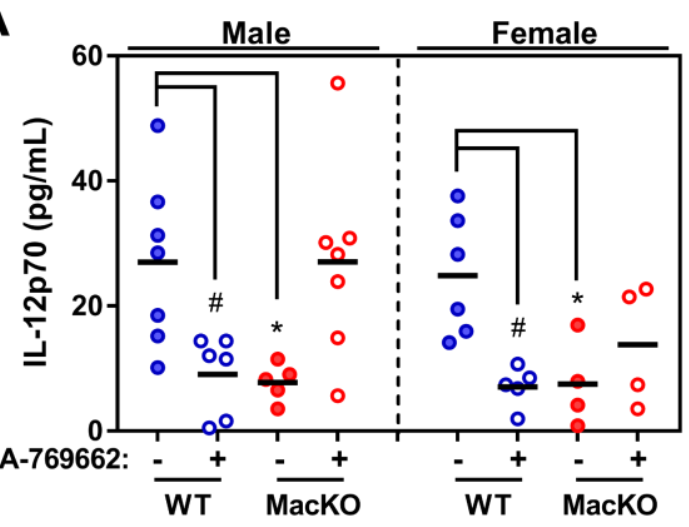

C

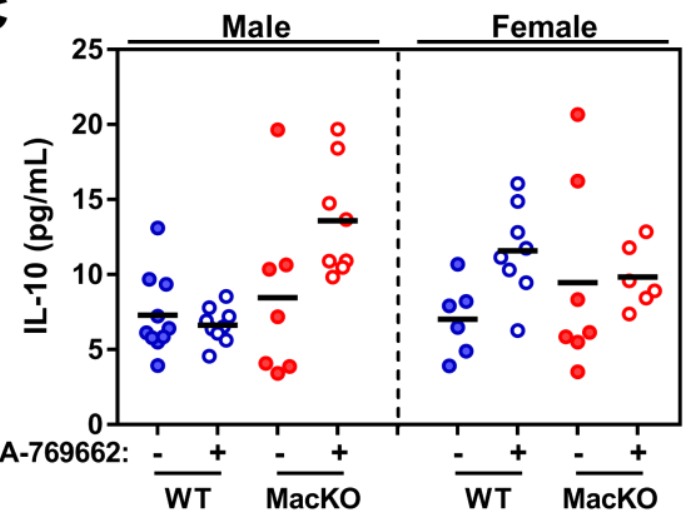

B

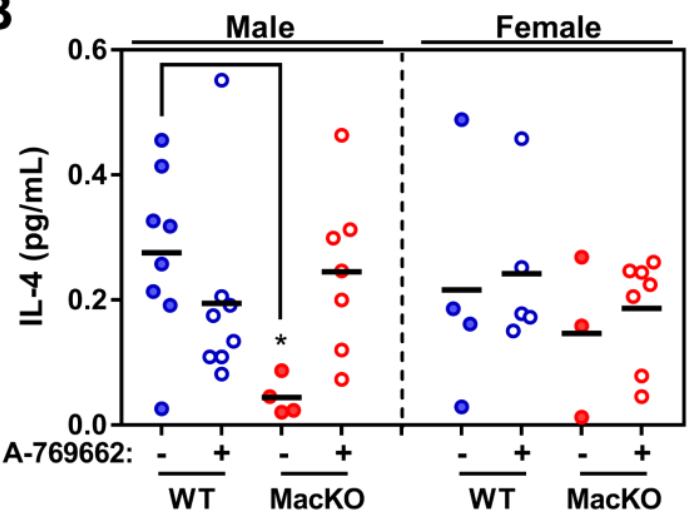

D

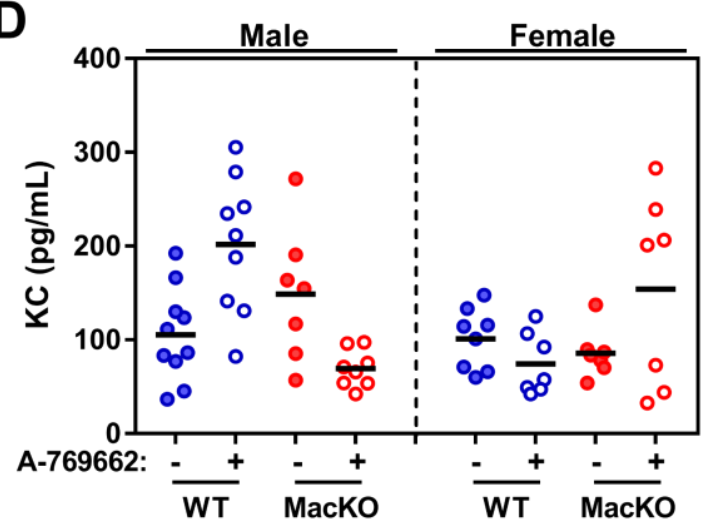

Figure 4. Myeloid AMPK signaling slightly alters systemic inflammation during the progression of atherosclerosis. Following WD-feeding, serum was collected to assess circulating inflammatory cytokines (A) IL-12p70, (B) IL-4, (C) IL-10, and (D) KC. Each data point represents the value from one animal ( $n=4-10 /$ group), where * represents $p<0.05$ between genotypes and \# is $p<0.05$ between treatments as calculated by a 2 way ANOVA. 

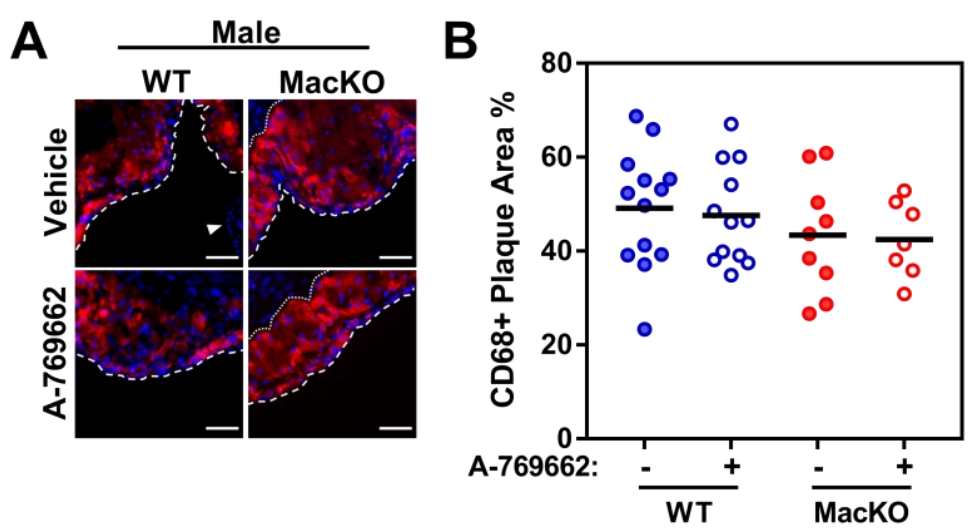

Figure 5. Myeloid AMPK signaling does not regulate the proportion of CD68-positive cells within the lesion but is implicated in total CD68 expression within male mice. (A) Representative images for immunofluorescent labeled lesions of male mice (CD68; Red, DAPI; Blue, where the luminal border of the lesion is denoted by white dashed and dotted line). (B) Quantification of CD68+ area as a percent of the total lesion area in lesions of male mice. Scale bar represents $50 \mu \mathrm{m}$. Each data point represents the mean value from one animal ( $n=7$-13/group). 

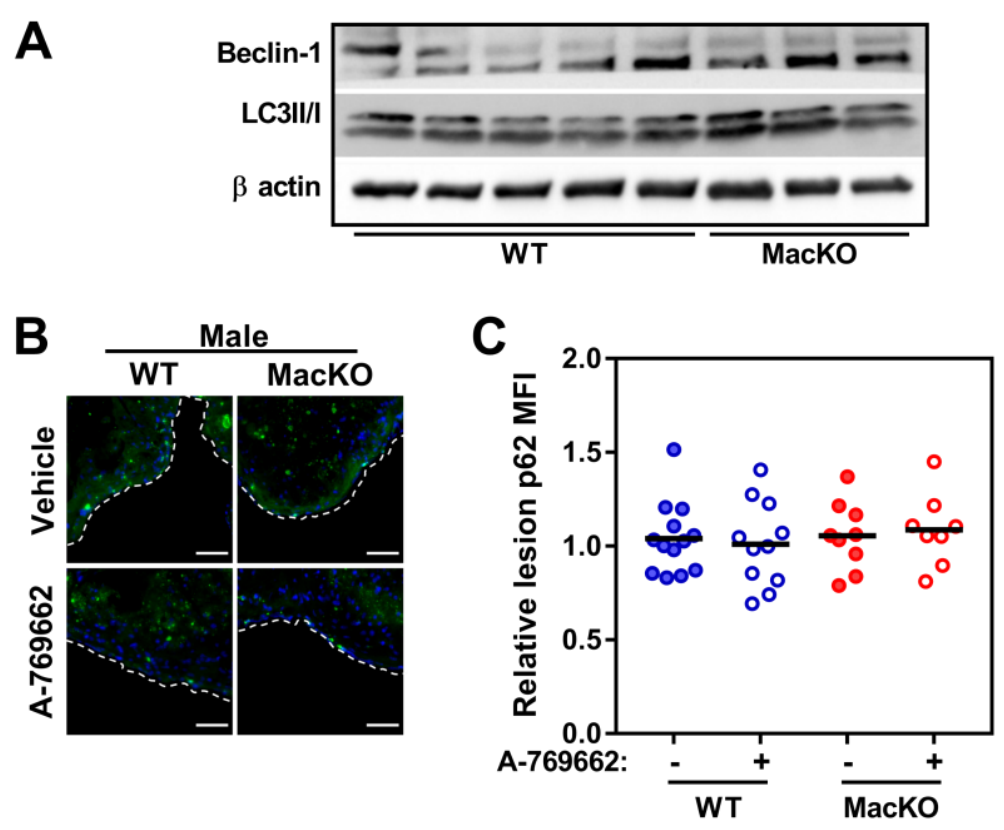

Figure 6. Myeloid AMPK signaling does not influence markers of lesion autophagy. (A) Whole aortic protein lysate from WT and MacKO (male) mice was probed for the expression of Beclin-1 and LC3II/1. (B) Close up representative image for immunofluorescent labeled lesions of male mice (p62; Green, DAPI; Blue, where the luminal border of the lesion is denoted by a white dashed line). (C) Quantification of the relative mean fluorescent intensity for p62 within the lesions of male mice. Scale bar represents $50 \mu \mathrm{m}$. Each data point represents the mean value from one animal ( $n=8-13$ /group). 